\title{
Approximate amenability for Banach sequence algebras
}

by

\author{
H. G. Dales (Leeds), R. J. Loy (Canberra) and \\ Y. ZHANG (Winnipeg)
}

\begin{abstract}
We consider when certain Banach sequence algebras $A$ on the set $\mathbb{N}$ are approximately amenable. Some general results are obtained, and we resolve the special cases where $A=\ell^{p}$ for $1 \leq p<\infty$, showing that these algebras are not approximately amenable. The same result holds for the weighted algebras $\ell^{p}(\omega)$.
\end{abstract}

1. Introduction. The concept of amenability for a Banach algebra $A$, introduced by Johnson in 1972 [7], has proved to be of enormous importance in Banach algebra theory (see [1], for example). In [3] several modifications of this notion were introduced; in this paper we shall focus on one of these, that of approximate amenability. We recall the definition in Definition 1.1 below.

Let $A$ be an algebra, and let $X$ be an $A$-bimodule. A derivation is a linear map $D: A \rightarrow X$ such that

$$
D(a b)=a \cdot D(b)+D(a) \cdot b \quad(a, b \in A) .
$$

For $x \in X$, set $\operatorname{ad}_{x}: a \mapsto a \cdot x-x \cdot a, A \rightarrow X$. Then $\operatorname{ad}_{x}$ is a derivation; these are the inner derivations.

Let $A$ be a Banach algebra, and let $X$ be a Banach $A$-bimodule. A continuous derivation $D: A \rightarrow X$ is approximately inner if there is a net $\left(x_{\alpha}\right)$ in $X$ such that

$$
D(a)=\lim _{\alpha}\left(a \cdot x_{\alpha}-x_{\alpha} \cdot a\right) \quad(a \in A),
$$

so that $D=\lim _{\alpha} \operatorname{ad}_{x_{\alpha}}$ in the strong-operator topology of $\mathcal{B}(A)$.

2000 Mathematics Subject Classification: Primary 46H20; Secondary 43A20.

Key words and phrases: amenability, approximate amenability, approximately inner, Banach sequence algebra.

The authors are grateful for support from the Royal Society of London and the Mathematical Sciences Research Visitors Program at the Australian National University (Dales), the London Mathematical Society Scheme 2 (Loy and Zhang), and NSERC grant 2389492001(Zhang). 
The dual of a Banach space $X$ is denoted by $X^{\prime}$; in the case where $X$ is a Banach $A$-bimodule, $X^{\prime}$ is also a Banach $A$-bimodule. For the standard dual module definitions, see [1].

Definition 1.1 ([3]). Let $A$ be a Banach algebra. Then $A$ is approximately amenable if, for each Banach $A$-bimodule $X$, every continuous derivation $D: A \rightarrow X^{\prime}$ is approximately inner.

The qualifier sequential prefixed to the above definition specifies that there is a sequence of inner derivations approximating the given continuous derivation.

We remark that, in [3], the notion of uniform approximate amenability was also introduced: a Banach algebra $A$ is said to be uniformly approximately amenable if, for each Banach $A$-bimodule $X$, each continuous derivation $D: A \rightarrow X^{\prime}$ is the limit of a sequence of inner derivations in the norm topology of $\mathcal{B}\left(A, X^{\prime}\right)$. In fact, it has recently been shown independently by Pirkovskii [10] and Ghahramani [4] that a uniformly approximately amenable Banach algebra is already amenable.

Of course, each amenable Banach algebra is approximately amenable. Some approximately amenable Banach algebras which are not amenable are constructed in [3]. For example, let $\left(A_{n}\right)$ be a sequence of unital, amenable Banach algebras. Then the sum $c_{0}\left(A_{n}\right)$ is always approximately amenable, but is not necessarily amenable [3, Example 6.1]. Further, it has been shown by Ghahramani and Stokke [5] that the Fourier algebra $A(G)$ is approximately amenable for each amenable, discrete group $G$, but it is known that $A(G)$ is not always amenable for an amenable group $G$ [9]. Examples of semigroup algebras of the form $\ell^{1}(S)$ that are approximately amenable but not amenable are given in [2]. Nevertheless there is something of a shortage of "natural" examples of approximately amenable Banach algebras which are not amenable.

In this paper, we shall consider when certain Banach sequence algebras on $\mathbb{N}$ are approximately amenable, a question left open in [3]. In particular, we shall consider the standard Banach sequence algebras $\ell^{p}=\ell^{p}(\omega)$, where $1 \leq p<\infty$ and $\omega$ is a weight on $\mathbb{N}$.

2. Basic constructions. When determining whether or not our Banach algebras are approximately amenable, we shall work from a characterization of approximately amenable Banach algebras which is a modification of that given in [3].

Let $A$ be Banach algebra. The projective tensor product $A \widehat{\otimes} A$ is a Banach $A$-bimodule under the operations defined by

$$
c \cdot a \otimes b=c a \otimes b, \quad a \otimes b \cdot c=a \otimes b c \quad(a, b, c \in A),
$$


and there is a continuous linear $A$-bimodule homomorphism $\pi: A \widehat{\otimes} A \rightarrow A$ such that $\pi(a \otimes b)=a b(a, b \in A)$ (see [1]).

Proposition 2.1. Let $A$ be a Banach algebra. Then $A$ is approximately amenable if and only if, for each $\varepsilon>0$ and each finite subset $S$ of $A$, there exist $F \in A \otimes A$ and $u, v \in A$ such that $\pi(F)=u+v$ and, for each $a \in S$ :

(i) $\|a \cdot F-F \cdot a+u \otimes a-a \otimes v\|<\varepsilon$;

(ii) $\|a-a u\|<\varepsilon$ and $\|a-v a\|<\varepsilon$.

Proof. Suppose that $A$ is approximately amenable. Then by [3, Corollary $2.2]$ there are nets $\left(M_{\alpha}\right)$ in $(A \widehat{\otimes} A)^{\prime \prime}$ and $\left(U_{\alpha}\right)$ and $\left(V_{\alpha}\right)$ in $A^{\prime \prime}$ such that, for each $a \in A$ :

(i) $a \cdot M_{\alpha}-M_{\alpha} \cdot a+U_{\alpha} \otimes a-a \otimes V_{\alpha} \rightarrow 0$;

(ii) $a-a \cdot U_{\alpha} \rightarrow 0$ and $a-V_{\alpha} \cdot a \rightarrow 0$;

(iii) $\pi^{\prime \prime}\left(M_{\alpha}\right)-U_{\alpha}-V_{\alpha} \rightarrow 0$.

(This corrects a typographical error in [3].) In each case convergence is in the $\|\cdot\|$-topology.

Let $Y$ denote the Banach space $(A \widehat{\otimes} A) \oplus A \oplus A \oplus A$. For each $a \in A$, define a convex set in $Y$ by setting

$$
\begin{aligned}
K_{a}:=\{(a \cdot m-m \cdot a+u \otimes a-a \otimes v, \\
\quad a-a u, a-v a, \pi(m)-u-v): m \in A \widehat{\otimes} A, u, v \in A\} .
\end{aligned}
$$

For the specified finite subset $S$ of $A$,

$$
K:=\prod\left\{K_{a}: a \in S\right\}
$$

is a convex set in the Banach space $Y^{S}$. The conditions above show that the weak closure of $K$ in $Y^{S}$ contains the zero element 0 of $Y^{S}$. By Mazur's theorem, it follows that 0 belongs to the $\|\cdot\|$-closure of $K$ in $Y^{S}$. Thus, with $\varepsilon>0$ as specified, there exist $F \in A \widehat{\otimes} A$ and $u, v \in A$ such that clauses (i) and (ii) of the proposition are satisfied and, further, such that $\|\pi(F)-u-v\|<\varepsilon$. By modifying $F$ and $u$ slightly, we may suppose, further, that $F \in A \otimes A$ and that $\pi(F)=u+v$.

Conversely, suppose that the condition in the proposition is satisfied. Consider the set $D:=(0,1) \times \mathcal{F}(A)$, where $\mathcal{F}(A)$ is the family of finite subsets of $A$, and order $D$ by setting

$$
\left(\varepsilon_{1}, S_{1}\right) \preccurlyeq\left(\varepsilon_{2}, S_{2}\right) \quad \text { whenever } \quad \varepsilon_{1} \geq \varepsilon_{2} \text { and } S_{1} \subseteq S_{2} .
$$

Then $(D, \preccurlyeq)$ is a directed set. The conditions show that there exist nets $\left(F_{\alpha}\right)$ in $A \widehat{\otimes} A$ and $\left(u_{\alpha}\right),\left(v_{\alpha}\right)$ in $A$, each indexed by $(D, \preccurlyeq)$, such that $\pi\left(F_{\alpha}\right)=$ $u_{\alpha}+v_{\alpha}$ and such that, for each $a \in A$, we have:

$$
\begin{gathered}
a \cdot F_{\alpha}-F_{\alpha} \cdot a+u_{\alpha} \otimes a-a \otimes v_{\alpha} \rightarrow 0 ; \\
a-a u_{\alpha} \rightarrow 0, \quad a-v_{\alpha} a \rightarrow 0 .
\end{gathered}
$$


Thus we have satisfied the conditions of [3, Corollary 2.2], and so $A$ is approximately amenable.

Corollary 2.2. Let $A$ be a Banach algebra with identity e. Then $A$ is approximately amenable if and only if, for each $\varepsilon>0$ and each finite subset $S$ of $A$, there exists $G \in A \otimes A$ with $\pi(G)=e$ and such that

$$
\|a \cdot G-G \cdot a\|<\varepsilon \quad(a \in S) .
$$

Proof. Suppose that such a $G$ exists, and set $u=v=e$ and $F=G+e \otimes e$. Then $\pi(F)=u+v$, and $F, u, v$ satisfy the conditions of Proposition 2.1.

Conversely, suppose that $F, u, v$ satisfy the above condition for a finite subset $S$ and with $\varepsilon / 3\|e\|$ replacing $\varepsilon$, and set

$$
G=F-u \otimes e-e \otimes v+e \otimes e .
$$

Then $\pi(G)=e$, and

$\|a \cdot G-G \cdot a\| \leq\|a \cdot F-F \cdot a+u \otimes a-a \otimes v\|+\|a-a u\|+\|a-v a\|<\varepsilon$, and so $A$ is approximately amenable by Proposition 2.1 .

For comparison, we recall [1], [8] that a Banach algebra $A$ is amenable if and only if there is a constant $C>0$ such that, for each $\varepsilon>0$ and each finite subset $S$ of $A$, there exists $F \in A \otimes A$ with $\|F\| \leq C$ such that, for each $a \in S$, we have:

(i) $\|a \cdot F-F \cdot a\|<\varepsilon$;

(ii) $\|a-a \pi(F)\|<\varepsilon$.

We remark that (ii) of Proposition 2.1 is exactly the condition that $A$ has both left and right approximate units [1, Definition 2.9.10]. We do not know whether or not an approximately amenable Banach algebra necessarily has (two-sided) approximate units.

We now give a variation of Proposition 2.1 in the case where $A$ is commutative. For each Banach algebra $A$, there is an isometry $\iota: A \widehat{\otimes} A \rightarrow A \widehat{\otimes} A$ such that $\iota(a \otimes b)=b \otimes a(a, b \in A)$.

Proposition 2.3. Let $A$ be a commutative Banach algebra. Then $A$ is approximately amenable if and only if, for each $\varepsilon>0$ and each finite subset $S$ of $A$, there exist $F \in A \otimes A$ with $\iota(F)=F$ and $u \in A$ such that $\pi(F)=2 u$, and, for each $a \in S$ :

(i) $\|a \cdot F-F \cdot a+u \otimes a-a \otimes u\|<\varepsilon$;

(ii) $\|a-a u\|<\varepsilon$.

Proof. Since $A$ is commutative,

$$
\iota(a \cdot F)=\iota(F) \cdot a \quad(a \in A, F \in A \widehat{\otimes} A) .
$$

Suppose that $A$ is approximately amenable, and take $\varepsilon>0$ and a finite subset $S$ of $A$. By Proposition 2.1, there exist $F, u$, and $v$ satisfying 
conditions (i) and (ii) of that result. For each $a \in S$, we have

$$
\|\iota(F) \cdot a-a \cdot \iota(F)+a \otimes u-v \otimes a\|<\varepsilon .
$$

Set $G=(F+\iota(F)) / 2$ and $w=(u+v) / 2$. Then $\iota(G)=G$ and $\pi(G)=2 w$. Further,

$$
\|a \cdot G-G \cdot a+w \otimes a-a \otimes w\|<\varepsilon \quad \text { and } \quad\|a-a w\|<\varepsilon .
$$

Thus the specified conditions are satisfied (with $w$ for $u$ ).

The converse is immediate.

3. Banach sequence algebras. We now introduce the specific algebras that will be considered in this paper. As usual $c_{00}$ will be the subalgebra of $\mathbb{C}^{\mathbb{N}}$ consisting of the sequences having finite support.

Definition 3.1. A Banach sequence algebra on $\mathbb{N}$ is a Banach algebra $A$ which is a subalgebra of $\mathbb{C}^{\mathbb{N}}$ such that $c_{00} \subset A$.

For example, $c_{0}=c_{0}(\mathbb{N})$ and $\ell^{p}=\ell^{p}(\mathbb{N})$ for $1 \leq p \leq \infty$ are Banach sequence algebras on $\mathbb{N}$.

Let $(A,\|\cdot\|)$ be a Banach sequence algebra on $\mathbb{N}$. Then

$$
\|a\| \geq|a|_{\mathbb{N}} \quad(a \in A)
$$

where $|\cdot|_{\mathbb{N}}$ denotes the uniform norm on $\mathbb{N}$. In the case where $c_{00}$ is dense in $A$, the algebra $A$ is natural on $\mathbb{N}$ [1, Proposition 4.1.35].

Throughout we write $\delta_{i}$ for the characteristic function of $\{i\}$ for $i \in \mathbb{N}$, and set

$$
e_{n}=\sum_{i=1}^{n} \delta_{i} \quad(n \in \mathbb{N}),
$$

so that $\left(e_{n}\right) \subset c_{00} \subset A$. When convenient we identify $a \in A$ both as the sequence $\left(a_{i}\right)$ and as the formal sum $\sum_{i} a_{i} \delta_{i}$. We shall also identify $A \otimes A$ with a space of functions on $\mathbb{N} \times \mathbb{N}$ by setting

$$
(a \otimes b)(i, j)=a_{i} b_{j} \quad(a, b \in A, i, j \in \mathbb{N}) ;
$$

in particular, $\delta_{i} \otimes \delta_{j}=\delta_{(i, j)}$, the characteristic function of $\{(i, j)\}$, for $i, j \in \mathbb{N}$. We shall also sometimes write $F=\sum_{i, j} F(i, j) \delta_{(i, j)}$ for $F \in c_{00} \otimes c_{00}$. Note that

$$
(a \cdot F)(i, j)=a_{i} F(i, j), \quad(F \cdot a)(i, j)=a_{j} F(i, j) \quad(i, j \in \mathbb{N}),
$$

and that $\pi(F)=\sum_{i} F(i, i) \delta_{i}$.

Definition 3.2. Let $A$ be a Banach sequence algebra on $\mathbb{N}$, and let $a \in A$. For $F \in c_{00} \otimes c_{00}$, set

$$
\Delta_{a}(F)=a \cdot F-F \cdot a+\pi(F) \otimes a-a \otimes \pi(F) .
$$

Clearly $\Delta_{a}(F) \in c_{00} \otimes c_{00}$ whenever $a \in c_{00}$. 
Proposition 3.3. Let $A$ be a Banach sequence algebra with $c_{00}$ dense in $A$. Then $A$ is approximately amenable if and only if, for each $\varepsilon>0$ and each finite subset $S$ of $A$, there exists $F \in c_{00} \otimes c_{00}$ with $\iota(F)=F$ such that, for each $a \in S$ :

(i) $\left\|\Delta_{a}(F)\right\|<\varepsilon$;

(ii) $\|a-a \pi(F)\|<\varepsilon$.

Proof. Suppose that $A$ is approximately amenable, and take $\varepsilon>0$ and a finite subset $S$ of $A$. Let $F$ and $u$ be given by Proposition 2.3. Since $c_{00}$ is dense in $A$, the space $c_{00} \otimes c_{00}$ is dense in $A \otimes A$, and so we can replace $F$ by an element $G \in c_{00} \otimes c_{00}$ such that (i) and (ii) of that proposition remain true, with $v=\pi(G) / 2$ replacing $u$. Now replace $G$ by

$$
H=G+\sum_{i}\left(v_{i}-\pi(G)_{i}\right) \delta_{i} \otimes \delta_{i},
$$

noting that the number of non-zero summands in the above sum is finite. This does not affect clauses (i) or (ii) of Proposition 2.3, and now $\pi(H)=v$. Thus conditions (i) and (ii) of the current proposition are satisfied.

The converse is similar.

We shall later consider only Banach sequence algebras $A$ which are selfadjoint. In such a situation the map $a \mapsto \bar{a}$ is necessarily continuous on $A$. It follows that we may replace $F$ by $F+\bar{F}$, and so take $F$ to be real-valued. Similarly, we may also suppose that the elements of the "test sets" $S$ are real-valued.

Proposition 3.4. Let $A$ be a Banach sequence algebra. Suppose that there is $\eta>0$ such that, for each $\varepsilon>0$ and each finite subset $S$ of $A$, there exists $u \in c_{00}$ with

$$
\|u\| \geq \eta \quad \text { and } \quad\|a-a u\| \cdot\|u\|<\varepsilon .
$$

Then $A$ is approximately amenable.

Proof. Take $u$ as given by (3.1), with $\varepsilon$ replaced by $\varepsilon \eta / 2$. Set

$$
F=u \otimes u+\sum_{i}\left(u_{i}-u_{i}^{2}\right) \delta_{i} \otimes \delta_{i} .
$$

Then $\pi(F)=u$ and, for each $a \in S$, we have

$$
\|a \cdot F-F \cdot a-a \otimes u+u \otimes a\|=\|a u \otimes u-a \otimes u+u \otimes a-u \otimes a u\|<\varepsilon
$$

and $\|a-a u\|<\varepsilon$. By Proposition 3.3, $A$ is approximately amenable.

The converse is immediate.

More general forms of this result for Banach function algebras on discrete spaces can be shown by the same sort of argument; see, for example, [5, Proposition 3.16]. 
We make the conjecture that the sufficient condition in Proposition 3.4 is in fact also necessary for $A$ to be approximately amenable. Indeed, we do not know an example of a Banach sequence algebra which is approximately amenable, but which does not have a bounded approximate identity. It is also conceivable that each Banach sequence algebra $A$ such that $c_{00}$ is dense in $A$ and $A=A^{2}$ is approximately amenable.

Corollary 3.5. Let $A$ be a Banach sequence algebra such that $A$ has a bounded approximate identity contained in $c_{00}$. Then $A$ is sequentially approximately amenable.

Proof. It is standard that $A$ has a sequential bounded approximate identity, say $\left(u_{n}\right)$, in $c_{00}$ [1, Corollary 2.9.18], and satisfying $\inf _{n}\left\|u_{n}\right\| \geq 1$. Let $\left\{x_{n}: n \in \mathbb{N}\right\}$ be a countable dense subset of $A$. Then, for each $n \in \mathbb{N}$, there exists $i=i(n)$ such that $\left\|x_{j}-x_{j} u_{i(n)}\right\|<1 / n$ for $1 \leq j \leq n$. Following Proposition 3.4, we set

$$
F_{n}=u_{i(n)} \otimes u_{i(n)}+\sum_{j \in \mathbb{N}}\left(u_{i(n), j}-u_{i(n), j}^{2}\right) \delta_{j} \otimes \delta_{j} .
$$

Then, for each $a \in A$ and $\varepsilon>0$, we have

$$
\left\|a \cdot F_{n}-F_{n} \cdot a-a \otimes u_{i(n)}+u_{i(n)} \otimes a\right\|=2\left\|a u_{i(n)}-a\right\| \cdot\left\|u_{i(n)}\right\|<\varepsilon
$$

for $n$ sufficiently large. Thus $\left(F_{n}, u_{i(n)}\right)$ gives a sequence with the required properties of [3, Corollary 2.2]. The sequential variant of [3, Theorem 2.1] holds (with the same argument), and so $A$ is sequentially approximately amenable.

Special cases of the above corollary have been shown in [4], where it is also shown that the converse holds for certain Banach sequence algebras.

We wish to stress that the function $F$ specified in Proposition 3.3 must satisfy conditions (i) and (ii) for each finite collection $S$ of elements. The following shows that, for many Banach sequence algebras $A$, we can find $F$ to satisfy these conditions for each single element $a \in A$. Indeed, the Banach sequence algebra $\ell^{1}$ satisfies the conditions of Proposition 3.6 below, but we shall see that it is not approximately amenable. To determine whether or not such an algebra $A$ is approximately amenable, we must look at sets $S$ with at least two elements.

We introduce the following notation. Let $A$ be a Banach sequence algebra on $\mathbb{N}$. For each $a \in A$ and each finite or cofinite subset $T$ of $\mathbb{N}$, set

$$
P_{T}: a \mapsto \sum_{i}\left\{a_{i} \delta_{i}: i \in T\right\}, \quad A \rightarrow A .
$$

We also write $P_{n}=P_{\{1, \ldots, n\}}$ and $Q_{n}=I-P_{n}$ for $n \in \mathbb{N}$. The family $\mathcal{C}$ of cofinite subsets of $\mathbb{N}$ will be directed by reverse set inclusion. 
Proposition 3.6. Let $A$ be a Banach sequence algebra, and let $a \in A$. Suppose that

$$
\lim \left\{\left\|P_{C} a\right\|: C \in \mathcal{C}\right\}=0 .
$$

Then, for each $\varepsilon>0$, there exists $F \in c_{00} \otimes c_{00}$ such that

$$
\left\|\Delta_{a}(F)\right\|<\varepsilon \text { and }\|a-a \pi(F)\|<\varepsilon .
$$

Proof. Let $\left\{B_{i}: i \in \mathbb{Z}^{+}\right\}$be the partition of $\mathbb{N}$ such that $a$ takes the constant value $a_{i}$ on $B_{i}$ for $i \in \mathbb{N}$, the value 0 on $B_{0}$, and $a_{i} \neq a_{j}$ whenever $i, j \in \mathbb{Z}^{+}$and $i \neq j$. Note that, by (3.2), each $B_{i}$ for $i \in \mathbb{N}$ is finite. For $n \in \mathbb{N}$, set

$$
D_{n}=\bigcup_{i=1}^{n} B_{i} \text { and } E_{n}=\bigcup_{i=n+1}^{\infty} B_{i},
$$

and set $\mu(n)=\min E_{n}$, so that $\mu(n) \rightarrow \infty$ as $n \rightarrow \infty$.

Fix $\varepsilon>0$, and take $n_{0} \in \mathbb{N}$ such that $\left\|P_{C} a\right\|<\varepsilon$ for each cofinite subset $C$ of $\mathbb{N}$ with $\min C \geq n_{0}$. Next choose $n_{1} \in \mathbb{N}$ such that $\mu\left(n_{1}\right) \geq n_{0}$. Set $C=E_{n_{1}} \cup\left(B_{0} \cap\left[n_{0}, \infty\right)\right)$, so that $D_{n_{1}}$ is finite and $C$ is cofinite with $\min C \geq n_{0}$. Take $u$ to be the characteristic function of $D_{n_{1}}$, so that

and hence

$$
a-a u=a \chi_{\mathbb{N} \backslash D_{n_{1}}}=P_{C} a,
$$

$$
\|a-a u\|=\left\|P_{C} a\right\|<\varepsilon .
$$

By (3.2), we may choose $m_{0} \in \mathbb{N}$ with $m_{0}>n_{1}$ and such that

$$
\left|D_{n_{1}}\right| \cdot\left\|Q_{m_{0}} a\right\|<\varepsilon / 2 .
$$

Now define $F$ as follows:

(a) For $j, k \leq n_{1}$, set

$$
F=1 \text { on } B_{j} \times B_{k} ;
$$

(b) for $j \leq n_{1}$ and $n_{1}<k \leq m_{0}$, set

$$
F=\frac{-a_{k}}{a_{j}-a_{k}} \text { on } B_{j} \times B_{k} ;
$$

(c) by symmetry for $k \leq n_{1}$ and $n_{1}<j \leq m_{0}$; and

(d) at remaining points, $F=0$.

Note that $u \in c_{00}, F \in c_{00} \otimes c_{00}$, and $\pi(F)=u$. Set $\Delta_{a}=\Delta_{a}(F)$.

Clearly $\Delta_{a}$ is zero except on the sets $\left(B_{j} \times B_{k}\right) \cup\left(B_{k} \times B_{j}\right)$ where $j \leq n_{1}$ and $k>m_{0}$. On the set

$$
\left(\bigcup_{j \leq n_{0}} B_{j}\right) \times\left(\bigcup_{k>m_{0}} B_{k}\right)
$$

we see that $a \cdot F-F \cdot a$ and $a \otimes u$ are zero, and that $u \otimes a=u \otimes Q_{m_{0}} a$. A similar formula holds when $j$ and $k$ are interchanged. Note that $Q_{m_{0}} a \otimes u$ 
and $u \otimes Q_{m_{0}} a$ have disjoint supports in $\mathbb{N} \times \mathbb{N}$. Thus

$$
\begin{aligned}
\left\|\Delta_{a}\right\| & =2\left\|Q_{m_{0}} a \otimes u\right\|=2\left\|\sum\left\{Q_{m_{0}} a \otimes \delta_{r}: r \in D_{n_{1}}\right\}\right\| \\
& \leq 2\left|D_{n_{1}}\right| \cdot\left\|Q_{m_{0}} a\right\|<\varepsilon
\end{aligned}
$$

by (3.4). This establishes (3.3).

Note the explicit dependence of $F$ on the element $a$ in clause (b) above. One is tempted to try the "more obvious" definition

$$
F_{i, j}= \begin{cases}1 & (i, j \leq n) \\ 0 & \text { (otherwise) }\end{cases}
$$

for suitably large $n \in \mathbb{N}$, so that $\pi(F)=e_{n}$. In this case, $F$ is independent of $a$. Suppose that $S$ is a finite subset of $c_{00}$ (rather than $A$ ). Then our function $F$ satisfies (i) and (ii) of Proposition 3.3 for each $a \in S$ (for sufficiently large $n \in \mathbb{N}$ ). However, this choice of $F$ does not work for all $a \in A$. For example, take $A=\ell^{1}$, and set $a=\sum_{j} j^{-3 / 2} \delta_{j} \in A$. Then

$$
\left\|\Delta_{a}\right\|=\sum_{j=n+1}^{\infty} j^{-3 / 2}\left\|\delta_{j} \otimes e_{n}-e_{n} \otimes \delta_{j}\right\|=2 n \sum_{j=n+1}^{\infty} j^{-3 / 2} \geq 4
$$

for each $n \in \mathbb{N}$.

In fact, let $A=\ell^{1}$, and let $S$ be a finite subset of $A^{2}$. Then we claim that, for each $\varepsilon>0$, there exists $F \in A \otimes A$ such that (3.3) holds for each $a \in S$. This may add some credence to our conjecture that $A^{2}=A$ for an approximately amenable Banach sequence algebra.

To prove this claim, we first recall Pringsheim's theorem: for a decreasing sequence $\left(a_{i}\right) \in A$, one has $\lim _{i} i a_{i}=0$.

Now take $a=\left(a_{i}\right) \in A$ with $0 \leq a_{i} \leq 1(i \in \mathbb{N})$. Certainly $a_{i} \rightarrow 0$, and so there is a permutation $\sigma$ of $\mathbb{N}$ such that $a_{\sigma(j)} \leq a_{\sigma(i)}$ for $j \geq i$ in $\mathbb{N}$. Thus $i a_{\sigma(i)} \rightarrow 0$. Fix $\varepsilon \in(0,1)$, and take $n \in \mathbb{N}$ such that $j a_{\sigma(j)}<\varepsilon / 2$ for $j \geq n$ and also $\sum_{j=n+1}^{\infty} a_{j}<\varepsilon$. Set $B=\sigma^{-1}\left(\mathbb{N}_{n}\right) \cup \mathbb{N}_{n}$, where $\mathbb{N}_{n}=\{1, \ldots, n\}$. Then set $u=\chi_{B}$, the characteristic function of $B$,

$$
F_{i, j}= \begin{cases}1 & (i, j \in B) \\ 0 & \text { (otherwise) }\end{cases}
$$

and $F=\sum_{i, j} F_{i, j} \delta_{(i, j)}$, so that $\pi(F)=u$. We see that

$$
s:=|B| \sum\left\{a_{i}^{2}: i \in \mathbb{N} \backslash B\right\} \leq 2 n \sum_{j=n+1}^{\infty} a_{\sigma(j)}^{2} \leq \frac{n \varepsilon^{2}}{2} \sum_{j=n+1}^{\infty} j^{-2}<\varepsilon .
$$

Thus

$$
\left\|a^{2} \cdot F-F \cdot a^{2}+u \otimes a^{2}-a^{2} \otimes u\right\|=2\left\|Q_{B} a^{2}\right\|\|u\|=s<\varepsilon,
$$


and we have built in the fact that $\left\|a^{2}-u a^{2}\right\|<\varepsilon$. It follows that the conditions of (3.3) are satisfied for $a^{2}$.

For finitely many elements in $A^{2}$, it suffices to consider the case where each of them is real-valued, and hence we need only consider differences of finitely many squares of non-negative elements of $A$, say the elements are $a^{(1)}, \ldots, a^{(k)}$. We then have finitely many permutations $\sigma_{1}, \ldots, \sigma_{k}$ of $\mathbb{N}$ that respectively render each of these latter sequences decreasing. We argue as above, with $n \in \mathbb{N}$ chosen so that, for each $1 \leq i \leq k$, we have $j a_{\sigma_{i}(j)}^{(i)}<\varepsilon / 2 k$ for $j \geq n$ and also $\sum_{j=n+1}^{\infty} a_{j}^{(i)}<\varepsilon$. Finally, we set

$$
B=\mathbb{N}_{n} \cup \bigcup_{i=1}^{k} \sigma_{i}^{-1}\left(\mathbb{N}_{n}\right) .
$$

The above claim now follows.

4. Approximate amenability for $\ell^{p}$. Take $1 \leq p<\infty$. Then $\ell^{p}$ is a Banach sequence algebra, and $c_{00}$ is dense in $\ell^{p}$. These algebras are discussed in [1, Example 4.1.42].

It is well known that $\ell^{p}$ is weakly amenable, but not amenable. Clearly the sequence $\left(e_{n}\right)$ is an approximate identity for $\ell^{p}$ such that $\left\|e_{n}\right\|_{p}=$ $n^{1 / p}(n \in \mathbb{N})$. Certainly each $a \in \ell^{p}$ satisfies equation (3.2) above.

It is shown in [3, Example 6.3] that $\ell^{p}$ is not sequentially approximately amenable. In this section we show that $\ell^{p}$ is not approximately amenable.

To this end, some preliminaries and further notations are needed.

First, note that the map

$$
T: \ell^{p} \times \ell^{p} \rightarrow \ell^{p}(\mathbb{N} \times \mathbb{N}), \quad T(x, y)(i, j)=x_{i} y_{j},
$$

is bilinear with $\|T\|=1$, and so there is a map

$$
\widetilde{T}: \ell^{p} \widehat{\otimes} \ell^{p} \rightarrow \ell^{p}(\mathbb{N} \times \mathbb{N})
$$

with $\widetilde{T}(x \otimes y)=T(x, y)\left(x, y \in \ell^{p}\right)$ and $\|\widetilde{T}\|=1$. Let $H \in c_{00} \otimes c_{00}$. Then

$$
\sum_{i, j}|H(i, j)|^{p} \leq\|H\|^{p},
$$

where $\|H\|$ denotes the norm of $H$ in $\ell^{p} \widehat{\otimes}^{p}$. (Of course, equality holds in the case where $p=1$.)

Fix throughout $\gamma_{j}=1 / j(j+1)$ and set $\gamma=\left(\gamma_{j}\right)$. Note that $\gamma$ is positive, decreasing, and satisfies

$$
k \gamma_{k} \leq \sum_{j=k+1}^{\infty} \gamma_{j}
$$


Now let $\eta=\left(\eta_{j}\right) \in \ell^{1}$ be positive and decreasing, and define elements $a, b$ in $\ell^{p}$ by

$$
a=\sum_{j=1}^{\infty} \eta_{j}^{1 / p} \delta_{2 j-1}, \quad b=\sum_{j=1}^{\infty} \eta_{j}^{1 / p} \delta_{2 j} .
$$

We show that, for a suitable choice of $\eta$ and for a certain $\varepsilon>0$, there is no element $F \in c_{00} \otimes c_{00}$ such that both the following inequalities are true:

$$
\begin{gathered}
\left\|\Delta_{a}(F)\right\|+\left\|\Delta_{b}(F)\right\|<\varepsilon \\
\|a-\pi(F) a\|+\|b-\pi(F) b\|<\varepsilon
\end{gathered}
$$

It would then follow from Proposition 3.3 that $\ell^{p}$ is not approximately amenable.

Throughout, we set $u=\pi(F)$. As we remarked earlier, we may suppose that $F$ (and $u$ ) are real-valued.

We first make a small reduction. We may suppose that $\varepsilon<\eta_{1}^{1 / p}$. Now assume that $F$ satisfies (4.4), with $\varepsilon$ replaced by $\varepsilon / 2$. Then $\eta_{1}^{1 / p}\left(1-u_{1}\right)<$ $\eta_{1}^{1 / p} / 2$, and so $u_{1}>1 / 2$. By replacing $u$ and $F$ by $u / u_{1}$ and $F / u_{1}$, respectively, we find new elements $F \in c_{00} \otimes c_{00}$ and $u \in c_{00}$ such that $\pi(F)=u$ and

$$
u_{1}=1 \quad \text { and } \quad\left\|\Delta_{a}(F)\right\|+\left\|\Delta_{b}(F)\right\|<\varepsilon .
$$

Thus we may always suppose that $u_{1}=1$.

We shall need to estimate $\left\|\Delta_{x}\right\|=\left\|\Delta_{x}(F)\right\|$ for $x=a, b$, and for this we shall use (4.1). Thus we require lower bounds for $\left|\Delta_{x}(m, n)\right|$ for $m, n \in \mathbb{N}$.

First consider the points $(2 i-1,2 j)$, where $i, j \in \mathbb{N}$. For convenience, define $s=F_{2 i-1,2 j}$. We calculate the values

$$
\begin{aligned}
& \Delta_{a}(2 i-1,2 j)=\eta_{i}^{1 / p}\left(s-u_{2 j}\right), \\
& \Delta_{b}(2 i-1,2 j)=\eta_{j}^{1 / p}\left(u_{2 i-1}-s\right) .
\end{aligned}
$$

In the case where $i \leq j$, so that $\eta_{i} \geq \eta_{j}$, geometrical considerations show that

$$
\left|s-u_{2 j}\right|^{p} \eta_{i}+\left|u_{2 i-1}-s\right|^{p} \eta_{j} \geq \eta_{j}\left(\left|u_{2 i-1}-u_{2 j}\right| / 2\right)^{p} .
$$

In a similar manner, the points $(2 i, 2 j-1)$ taken with $i \leq j-1$ and $j \geq 2$, so that $\eta_{i} \geq \eta_{j}$, lead to the estimate

$$
\left|t-u_{2 j-1}\right|^{p} \eta_{i}+\left|u_{2 i}-t\right|^{p} \eta_{j} \geq \eta_{j}\left(\left|u_{2 i}-u_{2 j-1}\right| / 2\right)^{p},
$$

where $t=F_{2 i, 2 j-1}$. 
[At the points $(2 i-1,2 j-1)$ and $(2 i, 2 j)$, where $i, j \in \mathbb{N}$, we have

$$
\begin{aligned}
\Delta_{a}(2 i-1,2 j-1) & =\left(\eta_{i}^{1 / p}-\eta_{j}^{1 / p}\right) F_{2 i-1,2 j-1}-\eta_{i}^{1 / p} u_{2 j-1}+\eta_{j}^{1 / p} u_{2 i-1}, \\
\Delta_{b}(2 i, 2 j) & =\left(\eta_{i}^{1 / p}-\eta_{j}^{1 / p}\right) F_{2 i, 2 j}-\eta_{i}^{1 / p} u_{2 j}+\eta_{j}^{1 / p} u_{2 i}, \\
\Delta_{a}(2 i, 2 j) & =\Delta_{b}(2 i-1,2 j-1)=0 .
\end{aligned}
$$

Since $\eta_{i} \neq \eta_{j}$ for $i \neq j$, there are choices of the values of $F$ at the points $(2 i-1,2 j-1)$ and $(2 i, 2 j)$ giving zero values to both $\Delta_{a}$ and $\Delta_{b}$ at all these points. We shall not use this fact.]

For $u=\left(u_{i}\right) \in c_{00}$, set

$$
\Phi_{p}(\eta, u)=\sum_{j=1}^{\infty} \eta_{j} \sum_{i=1}^{j}\left|u_{2 i-1}-u_{2 j}\right|^{p}+\sum_{j=2}^{\infty} \eta_{j} \sum_{i=1}^{j-1}\left|u_{2 i}-u_{2 j-1}\right|^{p} .
$$

It follows from (4.1), the above estimates, and the simple inequality

$$
(\|\alpha\|+\|\beta\|)^{p} \geq\|\alpha\|^{p}+\|\beta\|^{p} \quad(\alpha, \beta \in \mathbb{C}),
$$

that

$$
2^{p}\left(\left\|\Delta_{a}(F)\right\|+\left\|\Delta_{b}(F)\right\|\right)^{p} \geq \Phi_{p}(\eta, u)
$$

Set

$$
\theta_{p}(\eta)=\inf \left\{\Phi_{p}(\eta, u): u \in c_{00}, u_{1}=1\right\} .
$$

We seek to show that, for suitable choice of $\eta$, we have $\theta_{p}(\eta)>0$, for then (4.3) fails for any $\varepsilon$ with $0<\varepsilon<\min \left\{\theta_{p}(\eta)^{1 / p}, \eta_{1}^{1 / p}\right\} / 2$, and so $\ell^{p}$ is not approximately amenable.

We note that $\Phi_{p}(\eta, u)$ is reduced if every value of $u_{i}$ outside $[0,1]$ is replaced by its nearest neighbour in $[0,1]$. Thus we may suppose throughout that

$$
0 \leq u_{i} \leq 1 \quad(i \in \mathbb{N})
$$

For $d \geq 2$, consider the set

$$
S_{d}=\left\{u \in c_{00}: u_{1}=1, u_{i} \in[0,1](i=1, \ldots, d), u_{i}=0(i>d)\right\} .
$$

Certainly

$$
\alpha_{d}=\min \left\{\Phi_{p}(\eta, u): u \in S_{d}\right\}>0,
$$

and this minimum is attained. The question is whether or not

$$
\lim _{d \rightarrow \infty} \alpha_{d}>0 \text {. }
$$

Suppose for the moment that $p=1$, and take $\eta=\gamma$. Thus, in this case, $\Phi_{1}(\eta, u)$ from (4.5) becomes

$$
\Phi_{1}(\gamma, u)=\sum_{j=1}^{\infty} \gamma_{j} \sum_{i=1}^{j}\left|u_{2 i-1}-u_{2 j}\right|+\sum_{j=2}^{\infty} \gamma_{j} \sum_{i=1}^{j-1}\left|u_{2 i}-u_{2 j-1}\right| .
$$


Consider the values of $\Phi_{1}(\gamma, u)$ for sequences $u \in S_{d}$, where $d \geq 2$. Indeed, take such a point $u$ with $u_{d}>0$. We claim that, by setting $u_{d}=0$, the value of $\Phi_{1}(\gamma, u)$ is reduced.

To establish this claim, first suppose that $d=2 k+1$ for some $k \in \mathbb{N}$. By the change specified, we first increase each term in the summand

$$
\gamma_{k+1} \sum_{i=1}^{k}\left|u_{2 i}-u_{2 k+1}\right|
$$

by at most $u_{2 k+1} \gamma_{k+1}$, and so the sum itself increases by at most $k u_{2 k+1} \gamma_{k+1}$. On the other hand, we decrease the term

$$
\sum_{j=k+1}^{\infty} \gamma_{j}\left|u_{2 k+1}-u_{2 j}\right|=\left(\sum_{j=k+1}^{\infty} \gamma_{j}\right) u_{2 k+1}
$$

by $u_{2 k+1}$ times the sum $\sum_{j=k+1}^{\infty} \gamma_{j}$ of the tail. Other terms are not affected. However, for each $k \in \mathbb{N}$, we have

$$
k \gamma_{k+1} \leq k \gamma_{k} \leq \sum_{j=k+1}^{\infty} \gamma_{j}
$$

by (4.2), and so, in total, the value of $\Phi_{1}(\gamma, u)$ has been decreased.

Now suppose that $d=2 k$ for some $k \in \mathbb{N}$. By the change specified, we firstly increase each term in the summand

$$
\gamma_{k} \sum_{i=1}^{k}\left|u_{2 i-1}-u_{2 k}\right|
$$

by at most $u_{2 k} \gamma_{k}$, and so the sum itself increases by at most $k u_{2 k} \gamma_{k}$. On the other hand, we decrease the term

$$
\sum_{j=k+1}^{\infty} \gamma_{j}\left|u_{2 k}-u_{2 j-1}\right|
$$

by $u_{2 k}$ times the sum $\sum_{j=k+1}^{\infty} \gamma_{j}$ of the tail. Other terms are not affected. Once again, (4.2) ensures that the value of $\Phi_{1}(\gamma, u)$ has been decreased.

By continuing, we see that, subject to the constraints we have imposed, and in particular that $u \in c_{00}$ and $u_{1}=1$, the minimum value of $\Phi_{1}(\gamma, u)$ is attained at the point $v=(1,0,0, \ldots)$, and so

$$
\theta_{1}=\Phi_{1}(\gamma, v)=\sum_{j=1}^{\infty} \gamma_{j}=1
$$

Hence we obtain the required contradiction, at least in the case where $p=1$.

Now consider the case where $p>1$. Again we should like to show that $\theta_{p}(\eta)>0$ for suitable $\eta$. The above method for the case that $p=1$ does not now work; indeed, the minimum value $\min \left\{\Phi_{p}(\eta, u): u \in S_{d}\right\}$ need not 
occur at the point $u=(1,0,0, \ldots)$, and in fact, perhaps surprisingly, it does not necessarily occur at a decreasing sequence $u$ of $S_{d}$. In fact we cannot explicitly calculate $\theta_{p}(\eta)$, but we obtain a lower bound by the use of Hölder's inequality.

With $1 / p+1 / q=1$, choose $\alpha>0$ so small that $1-p \alpha / q>1 / 2$. Then we have

$$
\delta=\sum_{j=1}^{\infty} j \gamma_{j}^{1+\alpha}<\infty \text { and } \sum_{j=1}^{\infty} \gamma_{j}^{1-p \alpha / q}<\infty,
$$

and so, in particular, the formula $\eta_{j}=\gamma_{j}^{1-p \alpha / q}(j \in \mathbb{N})$ defines a sequence $\eta \in \ell^{1}$ which is positive and decreasing.

Note that

$$
\frac{1+\alpha}{q}+\left(1-\frac{p}{q} \alpha\right) \frac{1}{p}=\frac{p+q}{p q}=1
$$

and that $\gamma_{j}=\eta_{j}^{1 / p} \cdot \gamma_{j}^{(1+\alpha) / q}$. For each $u \in c_{00}$ with $u_{1}=1$ we apply Hölder's inequality to the sequence $\left(x_{r} y_{r}\right)$, where $\left(x_{r}\right)$ has generic term $\eta_{j}^{1 / p}\left|u_{2 i-1}-u_{2 j}\right|$ or $\eta_{j}^{1 / p}\left|u_{2 i}-u_{2 j-1}\right|$, and $\left(y_{r}\right)$ has the corresponding generic term $\gamma_{j}^{(1+\alpha) / q}$. Thus we obtain

$$
\begin{aligned}
1 \leq \Phi_{1}(\gamma, u) & =\sum_{j=1}^{\infty} \gamma_{j} \sum_{i=1}^{j}\left|u_{2 i-1}-u_{2 j}\right|+\sum_{j=2}^{\infty} \gamma_{j} \sum_{i=1}^{j-1}\left|u_{2 i}-u_{2 j-1}\right| \\
& \leq\left(\sum_{j=1}^{\infty} \sum_{i=1}^{j} \gamma_{j}^{1+\alpha}+\sum_{j=2}^{\infty} \sum_{i=1}^{j-1} \gamma_{j}^{1+\alpha}\right)^{1 / q} \Phi_{p}(\eta, u)^{1 / p} \\
& \leq(2 \delta)^{1 / q} \Phi_{p}(\eta, u)^{1 / p} .
\end{aligned}
$$

It follows that $\theta_{p}(\eta) \geq(2 \delta)^{-p / q}>0$, as required.

Thus we have the following result.

THEOREM 4.1. The Banach sequence algebras $\ell^{p}(\mathbb{N}), 1 \leq p<\infty$, are not approximately amenable.

It is immediate that $\ell^{p}(S)$ is not approximately amenable for any infinite set $S$, since there is a continuous epimorphism $\ell^{p}(S) \rightarrow \ell^{p}(\mathbb{N})$.

Take $1 \leq p<\infty$. In [3, Corollary 7.1] it was shown that the Banach algebras $\ell^{p}$ are essentially amenable, that is, any derivation into the dual of a neo-unital bimodule is inner. From Theorem 4.1 we conclude that essential amenability does not imply approximate amenability. It also follows by the Plancherel theorem that $L^{2}(\mathbb{T})$ fails to be approximately amenable, though by [6, Theorem 4.5] it is pseudo-contractible, that is, it admits a central (unbounded) approximate diagonal.

We finally consider a weighted variant of the $\ell^{p}$ algebras. 
Let $\omega \in[1, \infty)^{\mathbb{N}}$. For $p \geq 1$, we consider

$$
\ell^{p}(\omega)=\left\{f \in \mathbb{C}^{\mathbb{N}}: f \cdot \omega \in \ell^{p}\right\}
$$

where $f \cdot \omega$ denotes the sequence with the $i$ th coordinate $(f \cdot \omega)(i)=f_{i} \omega_{i}$ $(i \in \mathbb{N})$. With the norm

$$
\|f\|_{p, \omega}=\|f \cdot \omega\|_{p} \quad\left(f \in \ell^{p}(\omega)\right)
$$

$\ell^{p}(\omega)$ is a Banach algebra under pointwise operations. As previously, the $\operatorname{map} T: \ell^{p}(\omega) \times \ell^{p}(\omega) \rightarrow \ell^{p}(\omega \otimes \omega)=\ell^{p}(\omega \otimes \omega, \mathbb{N} \times \mathbb{N})$ given by

$$
T(x, y)(i, j)=x_{i} y_{j} \quad\left(x, y \in \ell^{p}(\omega), i, j \in \mathbb{N}\right)
$$

defines a contractive operator $\widetilde{T}: \ell^{p}(\omega) \widehat{\otimes} \ell^{p}(\omega) \rightarrow \ell^{p}(\omega \otimes \omega)$, where $\omega \otimes \omega$ denotes the weight on $\mathbb{N} \times \mathbb{N}$ such that $\omega \otimes \omega(i, j)=\omega_{i} \omega_{j}(i, j \in \mathbb{N})$. As for the case of $\ell^{p}$, we aim to show that for some $\varepsilon>0$ and elements $a, b \in \ell^{p}(\omega)$, there is no $F \in c_{00} \otimes c_{00}$ such that both the following inequalities are true:

$$
\begin{gathered}
\left\|\Delta_{a}(F)\right\|_{p, \omega \otimes \omega}+\left\|\Delta_{b}(F)\right\|_{p, \omega \otimes \omega}<\varepsilon \\
\|a-\pi(F) a\|_{p, \omega}+\|b-\pi(F) b\|_{p, \omega}<\varepsilon .
\end{gathered}
$$

We take $\gamma=\left(\gamma_{i}\right)$ and $\eta=\left(\eta_{i}\right)$ the same as in the proof of Theorem 4.1. Set

$$
\eta_{j}^{\prime}=\frac{\eta_{j}}{\omega_{2 j-1}^{p}}, \quad \eta_{j}^{\prime \prime}=\frac{\eta_{j}}{\omega_{2 j}^{p}} \quad(j \in \mathbb{N}),
$$

and define

$$
a=\sum_{j=1}^{\infty}\left(\eta_{j}^{\prime}\right)^{1 / p} \delta_{2 j-1}, \quad b=\sum_{j=1}^{\infty}\left(\eta_{j}^{\prime \prime}\right)^{1 / p} \delta_{2 j},
$$

so that $a, b \in \ell^{p}(\omega)$. Now for $F \in c_{00} \otimes c_{00}$ and $u=\pi(F)$, following the same argument as in the proof of Theorem 4.1, we find that

$$
2^{p}\left(\left\|\Delta_{a}(F)\right\|_{p, \omega \otimes \omega}+\left\|\Delta_{b}(F)\right\|_{p, \omega \otimes \omega}\right)^{p} \geq \Phi_{p}(\eta, u),
$$

where $\Phi_{p}(\eta, u)$ is given by equation (4.5). This finally shows that the value of $\left\|\Delta_{a}(F)\right\|_{p, \omega \otimes \omega}+\left\|\Delta_{b}(F)\right\|_{p, \omega \otimes \omega}$ is bounded away from 0 as a function of $F \in c_{00} \otimes c_{00}$. We therefore conclude with the following theorem.

THEOREM 4.2. The Banach sequence algebras $\ell^{p}(\omega), 1 \leq p<\infty$, are not approximately amenable for any weight $\omega$.

\section{References}

[1] H. G. Dales, Banach Algebras and Automatic Continuity, London Math. Soc. Monographs 24, Clarendon Press, Oxford, 2000.

[2] H. G. Dales, A. T.-M. Lau and D. Strauss, Banach algebras on semigroups and their compactifications, preprint, 2006.

[3] F. Ghahramani and R. J. Loy, Generalized notions of amenability, J. Funct. Anal. 208 (2004), 229-260. 
[4] F. Ghahramani, R. J. Loy and Y. Zhang, Generalized notions of amenability, II, in preparation.

[5] F. Ghahramani and R. Stokke, Approximate and pseudo-amenability of $A(G)$, preprint, 2005.

[6] F. Ghahramani and Y. Zhang, Pseudo-amenable and pseudo-contractible Banach algebras, Math. Proc. Cambridge Philos. Soc., to appear.

[7] B. E. Johnson, Cohomology in Banach algebras, Mem. Amer. Math. Soc. 127 (1972).

[8] - Approximate diagonals and cohomology of certain annihilator Banach algebras, Amer. J. Math. 94 (1972), 685-698.

[9] -, Non-amenability of the Fourier algebra of a compact group, J. London Math. Soc. 50 (1994), 361-374.

[10] A. Yu. Pirkovskii, Approximate characterizations of projectivity and injectivity for Banach modules, Math. Proc. Cambridge Philos. Soc., to appear.

Department of Pure Mathematics

University of Leeds

Leeds LS2 9JT, UK

E-mail: garth@maths.leeds.ac.uk

Department of Mathematics

University of Manitoba

Winnipeg R3T 2N2, Canada

E-mail: zhangy@cc.umanitoba.ca
Mathematical Sciences Institute Australian National University Canberra, ACT 0200, Australia E-mail: rick.loy@maths.anu.edu.au

Received June 26, 2006

Revised version August 15, 2006 